\title{
Use of 3D mapping system for ablating an accessory pathway associated with coronary sinus diverticulum
}

\author{
Mohammad Paymard, Marc W. Deyell, Santabhanu Chakrabarti, Zachary W. Laksman, Jacob Larsen and \\ John A. Yeung-Lai-Wah* ${ }^{*}$
}

\begin{abstract}
Background: This is a rare and challenging case of Wolff-Parkinson-White syndrome due to a posteroseptal accessory pathway located in the coronary sinus diverticulum. It is often difficult to precisely locate this type of accessory pathway, and the ablation procedure could be associated with collateral damage to the neighbouring coronary arteries.
\end{abstract}

Case Presentation: The patient was a 49-year-old female with Wolff-Parkinson-White syndrome who was referred for catheter ablation. She had had a previous unsuccessful attempt at ablation and had remained symptomatic despite drug therapy. The pre-procedural cardiac computed tomography scan revealed the presence of a diverticulum in the proximal coronary sinus. Using an advanced three-dimensional cardiac mapping system, the electroanatomic map of the diverticulum was created. The accessory pathway potential was identified within the diverticulum preceding the ventricular insertion. The accessory pathway was then successfully ablated using radiofrequency energy.

Conclusion: We have demonstrated that the advanced three-dimensional cardiac mapping system plays a very important role in guiding clinicians in order to precisely locate and safely ablate this type of challenging accessory pathway.

Keywords: Coronary sinus diverticulum, Posteroseptal accessory pathway, Supraventricular tachycardia, WolffParkinson-White syndrome, Three-dimensional cardiac mapping, Catheter ablation

\section{Background}

Wolff-Parkinson-White (WPW) syndrome is effectively treated by ablating the culprit accessory pathway (AP). Although the acute success rate of AP ablation is generally considered high, the APs that are located in the posteroseptal area sometimes remain challenging. The posteroseptal space is an anatomically complex region comprised of several structures, including

*Correspondence: jayeung1@gmail.com

Heart Rhythm Services, Division of Cardiology, Department of Medicine, University of British Columbia, 1160 Burrard St, \#401, Vancouver, BC V6Z 2E8, Canada atrioventricular valves, the ostium of the coronary sinus (CS) and the middle cardiac vein. Rarely, posteroseptal APs are located in CS anomalies such as diverticula which makes them even more challenging to ablate effectively and safely. The CS diverticulum is a rare congenital anomaly that is described as a venous pouch located within the epicardial layers of the posterior superior process of the left ventricle, with a neck opening into the proximal of the CS [1]. The association between CS diverticulum and WPW syndrome was first described by Guiraudon et al. [1]. The wall of CS diverticulum contains muscle fibres connecting atria and ventricles that can potentially act as AP [2]. Among patients with 
posteroseptal APs, it has been estimated that 1.6-9\% of them are located within the CS diverticulum $[1,3]$. The catheter ablation of AP located in the CS diverticulum could be associated with thermal injury to the adjacent coronary artery [4].

Advanced three-dimensional cardiac mapping systems are routinely used to locate and ablate conventional APs located at atrioventricular valve annuli but the data on their role for CS diverticulum APs are limited. We present a case of CS diverticulum AP-related WPW syndrome for which we used the three-dimensional cardiac mapping system to guide us to locate and ablate the AP.

\section{Case presentation}

An otherwise healthy 49-year-old woman with a history of recurrent drug-refractory paroxysmal supraventricular tachycardia and previous unsuccessful ablation attempt was referred to our Centre.

The baseline electrocardiogram (EKG) showed sinus rhythm and ventricular pre-excitation consistent with a posteroseptal AP (Fig. 1a). The pre-procedural cardiac computed tomography scan revealed the presence a diverticulum in the CS. Interestingly, she also had a persistent left-sided superior vena cava. We used Surpreme ${ }^{\mathrm{TM}}$ JSN Quadripolar diagnostic catheters to perform the electrophysiologic study and Agilis NxT Steerable sheath (Abbott, St Paul, MN, the USA)

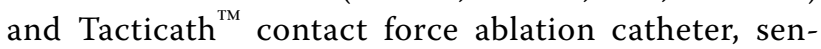
sor-enabled (Abbott, St Paul, MN, the USA) to perform radiofrequency ablation guided by an advanced three-dimensional cardiac mapping system (Abbott NavX). The anatomic map of the CS diverticulum was constructed. The diverticulum was attached to the CS body just beyond the point where the middle cardiac vein branched off from the CS.
Because the patient had manifest pre-excitation on surface EKG and also had a documented antidromic atrioventricular re-entry tachycardia during the previous attempt at ablation, we did not induce the clinical tachycardia this time but rather focussed on locating the AP. The AP was antegradely mapped during sinus rhythm as well as retrogradely during right ventricular pacing. The AP potential was identified within the diverticulum preceding the ventricular insertion (Fig. $1 \mathrm{~b}$ and c). This site was ablated by applying 20 watts of radiofrequency energy during which the preexcitation immediately resolved. At 12-month followup, the patient remained clinically asymptomatic and had no further palpitations.

\section{Discussion and Conclusions}

The ablation of manifest posteroseptal APs located in the CS diverticulum is challenging, and associated with a lower success rate and a higher chance of collateral injury. In order to tackle these issues, it is crucial to be able to precisely locate AP insertion sites by patiently mapping it antegradely during either sinus rhythm or atrial pacing, retrogradely during right ventricular pacing as well as trying to identify the AP potential if possible. This case report demonstrated that an advanced three-dimensional cardiac mapping system could be very useful and could facilitate the process of locating the AP and ablating it. The cardiac mapping system was used to create a detailed and accurate geometry of the CS and the diverticulum. Using colour-coded recording capability, the mapping system helped to identify the AP insertion sites accurately during pacing manoeuvres. The mapping system also minimised the total fluoroscopy time.

In conclusion, using an advanced cardiac mapping system has a very important role in precisely locating

(See figure on next page.)

Fig. 1 a Surface EKG shows ventricular pre-excitation with negative delta wave in limb lead II consistent with posteroseptal AP. b The AP potential can be seen between atrial and ventricular electrograms (long white arrow). The disappearance of the AP potential and loss of ventricular pre-excitation during RF ablation is demonstrated. The tip of ablation catheter is shown to be in the CS diverticulum where the earliest local ventricular EGM in relation to the delta wave was detected. c Retrograde mapping of the AP during ventricular pacing. The distal ablation catheter was in the CS diverticulum. The local atrial EGM detected on ablation catheter was earlier than atrial EGM on the HIS catheter. MCV: middle cardiac vein 
a

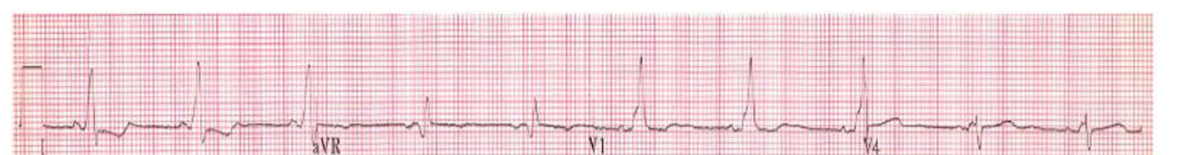

b

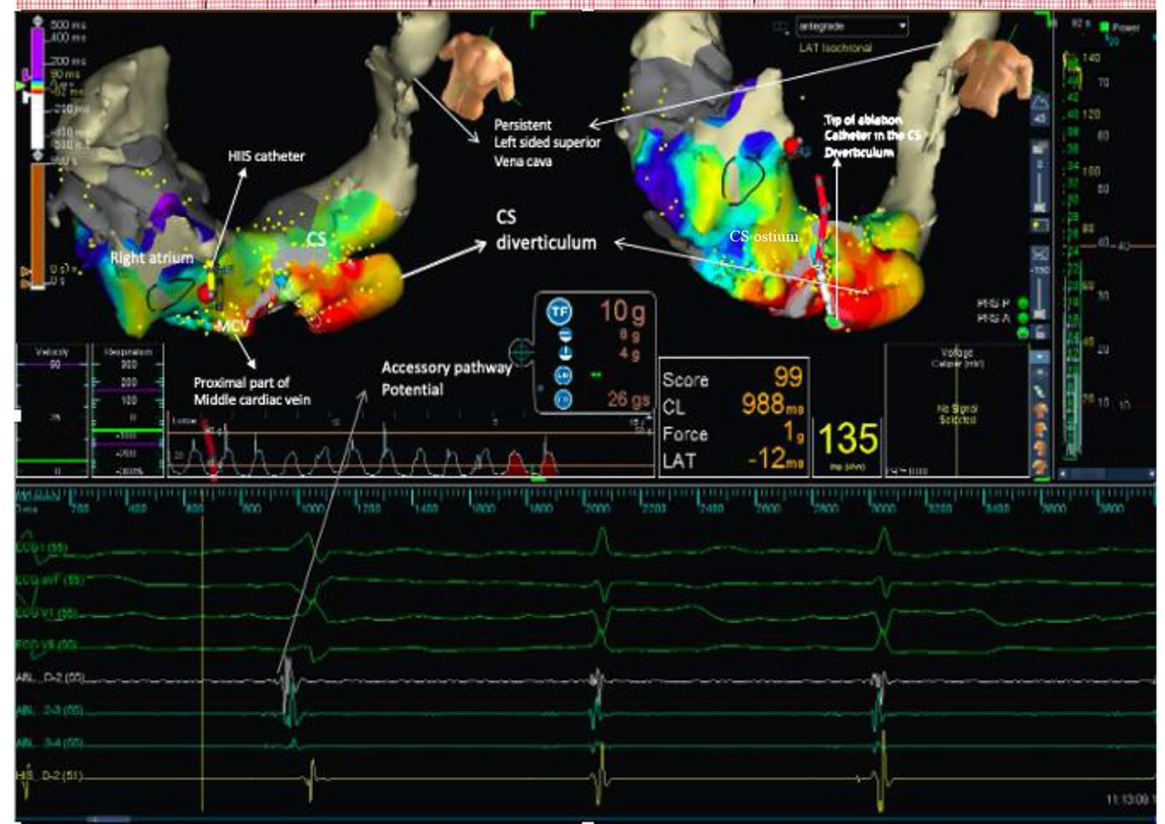

C

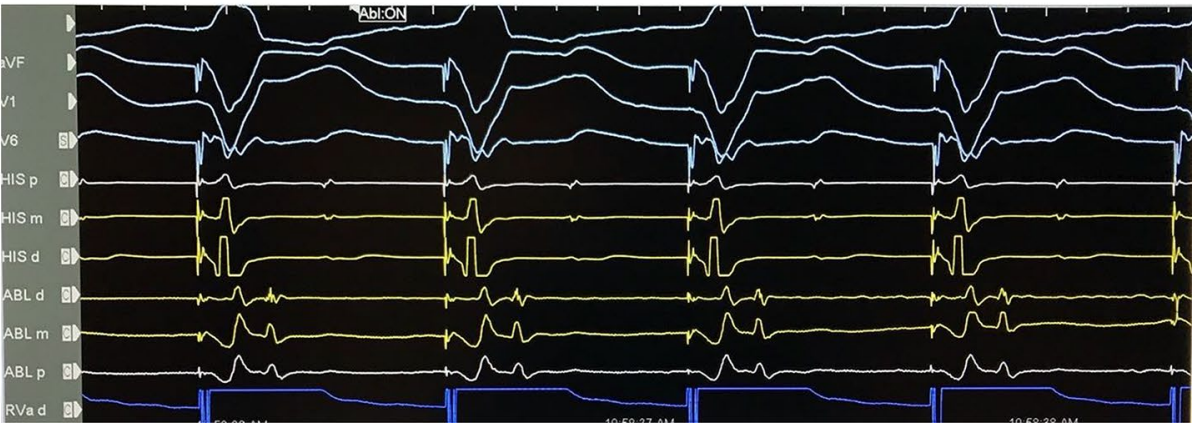


and safely ablating challenging posteroseptal APs located in the CS diverticulum.

\section{Abbreviations}

WPW: Wolff-Parkinson-White; AP: Accessory pathway; CS: Coronary sinus; EKG: Electrogram; MCV: Middle cardiac vein.

\section{Acknowledgments}

Donald Knapik, MSc

\section{Authors' contributions}

MP, MD, SC, ZL, JL, and JY all analyzed and interpreted the patient's data and involved in the patient's care. All authors read and approved the final manuscript.

\section{Availability of data and materials}

All data generated or analysed during this study are included in this published article.

\section{Declarations}

\section{Ethical Approval and Consent to participate}

Not applicable.

\section{Competing interests}

The authors declare that they have no competing interests.

\section{Consent for publication}

Written informed consent for publication of their clinical details and/or clinical images was obtained from the patient/parent/guardian/ relative of the patient. A copy of the consent form is available for review by the Editor of this journal.

Received: 19 February 2021 Accepted: 11 May 2021

Published online: 27 May 2021
References

1. Guiraudon GM, Guiraudon CM, Klein GJ, et al. The coronary sinus diverticulum: a pathologic entity associated with the Wolff-Parkinson-White syndrome. Am J Cardiol. 1988;62(10 Pt 1):733-5.

2. Lesh MD, Van Hare G, Kao AK, et al. Radiofrequency catheter ablation for Wolff-Parkinson-White syndrome associated with a coronary sinus diverticulum. Pacing Clin Electrophysiol. 1991;14(10):1479-84.

3. Leitz P, Wasmer K, Kobe J, et al. Remaining challenges in catheter ablation of accessory pathways: rare entity of coronary sinus diverticulum-associated pathways. Clin Res Cardiol. 2019;108(4):388-94.

4. Stavrakis $\mathrm{S}$, Jackman WM, Nakagawa $\mathrm{H}$, et al. Risk of coronary artery injury with radiofrequency ablation and cryoablation of epicardial posteroseptal accessory pathways within the coronary venous system. Circ Arrhythm Electrophysiol. 2014;7(1):113-9.

\section{Publisher's Note}

Springer Nature remains neutral with regard to jurisdictional claims in published maps and institutional affiliations.
Ready to submit your research? Choose BMC and benefit from:

- fast, convenient online submission

- thorough peer review by experienced researchers in your field

- rapid publication on acceptance

- support for research data, including large and complex data types

- gold Open Access which fosters wider collaboration and increased citations

- maximum visibility for your research: over $100 \mathrm{M}$ website views per year

At BMC, research is always in progress.

Learn more biomedcentral.com/submissions 